\title{
Las condiciones actuales de las universidades públicas en México
}

The current conditions of public universities in Mexico

\author{
Ana Laura Vargas Merino ${ }^{1}$ \\ Universidad Autónoma del Estado de Hidalgo
}

Recibido: 14.12 .2020

Aceptado: 28.12.2020

\section{Resumen}

El presente documento es parte de la construcción de un estado del conocimiento de la investigación titulada: Condiciones para la investigación educativa en Ciencias de la Educación. Un estudio comparado entre universidades públicas en México. La revisión documental se basó en la técnica de Mapeamiento Informacional Bibliográfico (MIB) que permitió procesar fuentes bibliográficas (Molina, et al, 2013) y poder identificar categorías analíticas, para esta comunicación se presentan los resultados de la categoría: Las condiciones actuales de las universidades públicas en México.

La organización de este documento es a través de dos ejes de análisis que le dan sentido: el primero de ellos, Políticas presupuestarias y distribución de recursos en las universidades públicas; y el segundo eje de análisis es sobre las universidades públicas como organizaciones administrativas y de gestión de la ciencia y la tecnología. Ambos ejes permiten contextualizar las condiciones a las que se enfrentan las universidades públicas, tanto en su naturaleza como

1va267114@uaeh.edu.mx https://orcid.org/0000-0003-0305-6897 https://scholar.google.com/citations?hl=es\&authuser=2\&user=lzNoCQkAAAAJ

Volumen 1. Número 2. Julio - Diciembre 2021 
instituciones autónomas, así como el papel que tienen en la administración de la ciencia. Se finaliza con las conclusiones y las rutas de trabajo a desarrollar.

Palabras clave: Universidad, Políticas presupuestarias, Administración de la ciencia.

\begin{abstract}
This document is part of the construction of a state of knowledge research entitled: Conditions for Educational Research in Educational Sciences. A comparative study among public universities in Mexico. The documentary review was based on the Bibliographic Information Mapping (MIB) technique that allowed to process bibliographic sources (Molina, et al, 2013) and to identify analytical categories, for this communication the results of the category are presented: The current conditions of public universities in Mexico.
\end{abstract}

The organization of this document is through two axes of analysis that give it meaning: the first of them, Budgetary policies and distribution of resources in public universities; and the second focus of analysis is on public universities as administrative and science and technology management organisations. Both axes allow to contextualize the conditions faced by public universities, both in their nature as autonomous institutions, as well as the role they have in the administration of science. It concludes with the conclusions and the work routes to develop.

Keywords: University; Budgetary policies; Science administration.

\title{
Introducción
}

El presente análisis: Las condiciones actuales de las universidades públicas en México, es parte de las primeras acciones para adentrarse en el tema de las universidades públicas como organizaciones que administran y gestionan la ciencia y la tecnología de este país. Se considera que es a través de la construcción de un estado del conocimiento, como una 
herramienta analítica y reconstructiva que ayude a identificar aquellos aspectos teóricos, situaciones problemáticas, metodologías y vacíos en el tema afín a este proyecto de investigación.

Se parte principalmente del Mapeamiento Informacional Bibliográfico (MIB) como una opción para localizar contenidos y procesar fuentes bibliográficas (Molina, et al, 2013), atendiendo rubros específicos que ayudan a determinar, desde una perspectiva sistémicodescriptiva, categorías de análisis, conceptos, tipos de fuentes e identificación de las ideas principales del autor.

Este documento está conformado por dos ejes de análisis que le dan sentido: el primero de ellos, Políticas presupuestarias y distribución de recursos en las universidades públicas, en el cual se deja ver que la asignación presupuestaria para estas instituciones públicas es un juego político que está mediado por el campo político mexicano, en el que intervienen sus características, como la centralización de recursos, por ejemplo; el segundo eje de análisis es sobre las universidades públicas como organizaciones administrativas y de gestión de la ciencia y la tecnología, en el que se indaga primero sobre la transformación de estas instituciones, después sobre el papel que tienen en el desarrollo de capacidades de investigación y de transferencia de conocimiento, para concluir con una breve revisión sobre los retos a los que se enfrentan. Por último, se presentan las conclusiones y las rutas de trabajo por desarrollar.

\section{Políticas presupuestarias y distribución de recursos en las universidades públicas}

El contexto que brinda la geopolítica como espacio-tiempo y como estructuras de poder proporciona referentes con respecto a las relaciones de Estado, pero también como herramienta que orienta la formulación de políticas para promover procesos transformativos en las estructuras de mercado y en la sociedad. Según la revisión documental, la economía del conocimiento no siempre proviene de un capitalismo homogéneo, aún en países desarrollados se van a encontrar diferencias entre las formas y grados de desarrollo (Lemus, 2018). En 
México no es la excepción, ya que son evidentes las diferencias económicas, a las cuales se suma la centralización del capital, agudizando aún más las diferencias.

Se ha identificado como problemática el pobre financiamiento en CIT (Ciencia, Innovación y Tecnología), principalmente del sector privado, que es 30\% menor en México en comparación con medidas internacionales, que llega a ser superior al 50\% del gasto (CONACYT, 2017), (OEI, 2018), siendo un inconveniente para el país, para poder avanzar hacia una economía del conocimiento capaz de vincular al sector público con las universidades públicas, los centros de investigación y con el sector privado principalmente.

Entonces ¿Cómo las universidades públicas pueden contribuir al desarrollo de una economía del conocimiento basada en una sociedad del conocimiento? El reto es difícil sobre todo por la influencia que puede generar con el marco de política en ciencia y tecnología en México. Para esta categoría Condiciones de las universidades públicas en México, la revisión de trabajos ayudó a identificar dos aspectos, el primero de ellos, Políticas presupuestarias y distribución de recursos en las universidades públicas, dejan ver para algunos autores como Zepeda $^{2}$ (2016) que la asignación presupuestaria para las universidades públicas es un juego político determinado por el partido político en el poder, en el Congreso y en los gobiernos estatales, los cuales influyen en la asignación de los recursos a favor o en contra. Afirmación que nos acerca a reflexionar sobre dos aspectos que maneja Bourdieu (2000, pág. 14), el primero sobre el juego político, siendo aquello que permita negociar un compromiso, hacer silencio sobre algún tema o proteger a los amigos de manera discreta, incluso mediar la relación con los medios de comunicación; el segundo aspecto, como un mecanismo para cerrar el campo político a los profanos (ajenos), que le provee autonomía con respecto a otros campos. Sin embargo, cuando el juego político excede los límites de su propio campo, puede afectar a otras instituciones.

\footnotetext{
${ }^{2}$ Se analizaron los subsidios a 32 universidades estatales en México por medio de regresión multivariada panel para el periodo 1990-2009.
} 
El trabajo de Zepeda (2016) pone en evidencia ese juego político, pero no logra llegar al punto de quiebre con respecto a la evolución del campo político en México, quedando de manera descriptiva lo que implica una transición política o la alternancia de poder. El autor encontró que "el gobierno federal premia a las universidades públicas estatales ubicadas en entidades gobernadas por partidos de oposición con quienes tiene más afinidad ideológica o posibilidades de negociación y castiga a las ubicadas en entidades gobernadas por partidos sin poder de veto legislativo e ideológicamente opuestos" (Zepeda Gil, 2016, p. 901). Esta premiación, a su vez, acrecienta el capital político de los agentes que intervienen y está determinado por el peso político de su partido (Bourdieu, 2000, pág. 18), demostrando los intereses políticos se sobreponen a la concepción de un Estado benefactor.

Otros trabajos como los de Arechavala \& Sánchez (2017) revelan un gobierno altamente centralizado sin importar qué partido está en el poder, siendo que la Ciudad de México concentra al menos el 39\% del presupuesto total para este sector educativo, siendo destinado a la Universidad Nacional Autónoma de México (UNAM), al Instituto Politécnico Nacional (IPN) y a la Universidad Autónoma Metropolitana (UAM). Los autores añaden que este hecho ha generado un desbalance con el resto de las instituciones en el país. Dicha condición se acentúa más, al privilegiar el financiamiento público sobre el financiamiento privado (Estado benefactor), contraponiéndose con el tan añorado aumento del PIB, que hasta la fecha no alcanza el 1\% para el desarrollo de ciencia y tecnología (OEI, 2018).

Sin embargo, Arechavala \& Sánchez (2017) no consideran que, universidades como la UNAM cuentan con una organización de sus sistemas científicos que orientan y determinan la puesta en marcha de políticas internas que propician condiciones para el desarrollo de investigación, ciencia y tecnología, tal y como lo demuestra Márquez (2011), justificando así un presupuesto más alto con respecto a otras universidades estatales.

La afirmación anterior lleva a reflexionar sobre la distribución de los recursos asignados a las universidades públicas, ya que al provenir del financiamiento público son para RodríguezGómez (2019), instituciones públicas del Estado y, como tales, pueden ser auditadas, 
supervisadas y evaluadas por las entidades autorizadas para ello para ello ${ }^{3}$. Lo que deja ver una tensión entre el presupuesto recibido y la autonomía, entendida esta última como la libertad para el uso de esos recursos económicos. Cabe señalar que, desde el 2017 se ha centrado la atención hacia la problemática de corrupción dentro de las universidades, debido al caso de La estafa maestra ${ }^{4}$, que involucra a varias universidades públicas con el desvío de recursos federales entre dependencias gubernamentales y proveedores privados, situación que genera debate sobre su autonomía y el uso de su presupuesto. Siendo que los aspectos sobre el presupuesto de las universidades públicas y su distribución deben ser profundizados, convirtiéndose en una ruta por seguir para esta investigación, a su vez nos abre paso para analizar a las universidades públicas como organizaciones administradoras de la ciencia y la tecnología en México.

\section{Las universidades públicas como organizaciones administrativas y de gestión de la ciencia y la tecnología}

Las universidades públicas pueden ser vistas como organizaciones administrativas, de gestión de la ciencia y la tecnología, siendo el segundo aspecto que compone esta categoría. Sin embargo, Arechavala \& Sánchez (2017) consideran que Latinoamérica y en particular México, ha iniciado tardíamente la transformación de sus universidades hacia el desarrollo de capacidades de investigación y de transferencia de conocimiento. Ya que las políticas de educación superior en la última década primero han estado encaminadas al incremento de la cobertura (matricula) y después para incrementar la calidad de sus programas educativos, además de lidiar con las exigencias para que generen más oportunidades de educación superior de carácter gratuito a la población (Rodríguez-Gómez, 2019), (Arechavala \& Sánchez, 2017). Sin embargo, Zepeda (2016) señala que, aún no se logra incrementar la cobertura, debido a que el presupuesto no es suficiente, en especial de aquellas que se encuentran en provincia;

\footnotetext{
${ }^{3}$ Sistema Nacional de Fiscalización, el Sistema Nacional Anticorrupción, ambos instituidos formalmente a través de la reforma al artículo 113 Constitucional en 2015, y el Sistema Nacional de Transparencia, derivado de un grupo de reformas de rango constitucional en enero de 2014 (Rodríguez-Gómez, 2019).

${ }^{4}$ Castillo, M., Roldán, N., \& Ureste, M. (2017, septiembre 5). La estafa maestra. Graduados en desaparecer dinero público. Recuperado 21 de mayo de 2020, de https://www.animalpolitico.com/estafa-maestra/
} 
este autor destaca que los recursos obtenidos, son usados en gasto corriente, sueldos de docencia y mantenimiento de las instalaciones, quedando fuera la inversión para la producción científica. Pese a esta realidad muchas universidades siguen desarrollando investigación a través de la gestión y búsqueda de recursos en otros programas gubernamentales, aunque aún no se atreven a buscar financiamiento privado, incluso es probable que existan candados desconocidos que pueden impedir que se lleven a cabo gestiones en este rubro.

Asociado al desarrollo de capacidades de investigación y de transferencia de conocimiento en las universidades públicas, se identifican problemas que aún imperan, como la separación de la docencia y la investigación, ya que cada una es realizada por un segmento distinto del personal académico con que cuentan las universidades. Sobre este tema Arechavala \& Sánchez (2017), señalan que la universidad pública debe abordar cuatro rubros para superar esta problemática: a) incentivar al personal académico con alto potencial para la investigación a través de la formación y la participación en eventos de divulgación científica; b) infraestructura: proporcionar áreas de trabajo adecuadas; c) promover programas de posgrado con madurez; y d) desarrollo de sistemas y procesos administrativos eficientes de apoyo a la investigación.

Aunado a toda la discusión ya planteada, la universidad pública se enfrenta a una carrera por el reconocimiento institucional a través de los rankings de universidades, que según Albornoz \& Osorio (2018) son un mercado de reconocimiento social, que ha adquirido gran visibilidad en el campo de las políticas de la educación superior y en las estrategias de su desarrollo institucional; su repercusión alcanza a los medios de comunicación en todo el mundo, se asocia con la cantidad de patentes y producciones científicas, convirtiéndose en mediciones globales con gran aceptación social. Cabe señalar que el trabajo de Albornoz \& Osorio (2018) hace una crítica a los rankings de universidades como sinónimo de calidad global y que orienta a las universidades a transformarse para la contienda interinstitucional.

Entonces, valdría la pena pensar más sobre la transformación de la universidad pública, como producto de múltiples y variadas condiciones sociohistóricas pasadas y presentes, que según 
Pacheco (2009) tiene vínculo con su condición de institución social, la cual ha variado en la medida en que los patrones más tradicionales tienen a debilitarse ante la presencia de las nuevas y más complejas demandas e intereses por atender. Bajo esa misma lógica Gibbons (citado en Valero, Molina \& Ponce 2019), sostiene que las universidades, tienen grandes retos ante los cambios actuales en la producción del conocimiento, empezando por la necesidad de una profunda transformación.

Algunos autores como Pérez Mora, Prieto \& Castellanos (2012) sostienen que la universidad pública está perdiendo su autonomía en relación con las fuerzas de mercado, sin embargo, no se está de acuerdo totalmente con esta aseveración, ya que, a nivel internacional, se señala la necesidad de mayor participación del sector privado, principalmente aquel que se ve beneficiado por la ciencia y la tecnología, reclamo encaminado a aquellas empresas internacionales que no invierten en este país. Sin embargo, en otras obras Pérez Mora se contradice principalmente cuando se asocia con la movilización del conocimiento ${ }^{5}$ (y la gestión), ya que señala que la investigación no debe ser exclusiva de las universidades, sino que debe ampliarse a las empresas, el sector gubernamental y la sociedad, en este sentido el conocimiento se vuelve un elemento que debe moverse entre múltiples instituciones y actores (Pérez Mora \& Inguanzo, 2018).

Aunque se está de acuerdo con lo establecido sobre (Pérez Mora \& Inguanzo, 2018):

La universidad y sus académicos enfrentan diversos retos para generar acciones que fortalezcan su autonomía frente a las políticas y discursos legitimadores de la economía del conocimiento. Las universidades deben trabajar a nivel de la gestión, nivel que se constituye como su ámbito de influencia, para promover la movilización del conocimiento (p. 79).

\footnotetext{
${ }^{5}$ La movilización del conocimiento abarca una amplia gama de actividades relacionadas con la producción y utilización de resultados de investigación, incluyendo la síntesis de conocimiento, difusión, transferencia, intercambio y co-creación o coproducción por investigadores y usuarios de conocimiento (Pérez Mora \& Inguanzo Arias, 2018).
} 
Las anteriores aseveraciones hacen pensar sobre la universidad pública, principalmente por su conformación orgánica, que privilegia su estatus de autonomía, y cómo en algunas, caso concreto la UNAM, la ciencia universitaria es valiosa para entender su organización, gestión y administración a través de sus sistemas de ciencia y tecnología, que determinan la puesta en marcha de políticas institucionales (Márquez, 2011). Por tanto, algunos autores como Pacheco (1994) optan por llamar a esta organización universitaria como un marco obligado para ubicar las condiciones que influyen en la actividad científica mexicana, como estructura institucional con carácter profesionalizante o profesionalización de la actividad científica ha permitido contextualizar la organización de la universidad. Al respecto se puede sumar que, en esta organización universitaria, los investigadores tienen un papel central como capital intelectual, por lo que conocer las condiciones en las cuales desarrollan su trabajo investigativo, es una ruta de trabajo pendiente para esta investigación.

\section{Conclusiones}

Para poder determinar las condiciones en las que operan las universidades públicas en México, es preciso conocer más sobre los ejes de análisis que nos brindó la revisión de investigaciones, de informes y documentos abiertos, por un lado, se parte de que el financiamiento en CIT es mayoritariamente público, del cual se alimenta la idea del Estado benefactor, siendo que el sector privado invierte poco en este rubro. Por otro lado, la asignación de presupuesto, la cual varía según se trate de una universidad pública de provincia o de una que se ubica en el centro del país. Como se identificó, algunos autores hablan de la existencia de un juego político que interviene en ese proceso, el cual puede privilegiar o castigar a dichas instituciones, entonces se observa que este juego sale de los límites del campo político, en el que los agentes que intervienen en ello van abarcando espacios que ya no pertenecen a ese espacio. Valdría la pena ahondar más en ese fenómeno.

Sin embargo, la evidencia obtenida en este documento no es suficiente, ya que algunas universidades públicas cuentan con un robusto sistema científico que orientan y determinan políticas internas que propician condiciones para el desarrollo de investigación, ciencia y 
tecnología, justificando así un presupuesto más alto con respecto a otras universidades estatales. Por lo que este tema se convierte en una ruta de trabajo a desarrollar en la investigación, pero sienta las bases sobre su complejidad, ya que de ello parte la organización administrativa y científica de estas instituciones, la cual está mediada por la autonomía como principal rasgo, el cual ha sido cuestionado en los últimos años.

La organización administrativa y científica de las universidades públicas, fue el segundo eje de análisis tratado en este trabajo, del cual se parte de la necesaria transformación que deben implementar hacia el desarrollo de capacidades de investigación y de transferencia de conocimiento. Se reconoce que en México este proceso ha sido tardío y desigual entre instituciones, sin embargo, es tema necesario que vuelve a poner sobre la mesa el tipo de financiamiento que reciben y los modos de ejercer la autonomía.

Por último, ambos ejes de análisis desarrollados en este documento brindan de manera general, las condiciones que enfrenta las universidades públicas como como organizaciones que proveen un marco de estructura institucional que proporciona las condiciones para que los investigadores desarrollen de manera profesional la investigación. Esta relación sistémica entre la universidad pública y el desarrollo de la investigación es una de las principales rutas a pendientes para el trabajo de investigación.

\section{Referencias}

Albornoz, M., \& Osorio, L. (2018). Rankings de universidades: calidad global y contextos locales. Revista Iberoamericana de ciencia, tecnología y sociedad, 13(37), 13-51. Recuperado de https://www.redalyc.org/jatsRepo/924/92457720006/html/index.html

Arechavala Vargas, R., \& Sánchez Cervantes, C. (2017). Las universidades públicas mexicanas: los retos de las transformaciones institucionales hacia la investigación y la transferencia de conocimiento. Revista de la Educación Superior, 46(184), 21-31. Recuperado de http://www.scielo.org.mx/pdf/resu/v46n184/0185-2760-resu-46-184-21.pdf 
Bourdieu, P. (2000). Sobre el Campo Político. Francia: Presses Universitaires de Lyon.

CONACYT. (2017). Informe General del estado de la ciencia, la tecnología y la innovación 2017. Ciudad de México: Consejo Nacional de Ciencia y Tecnología. Recuperado de http://www.siicyt.gob.mx/index.php/transparencia/informes-conacyt/informe-general-delestado-de-la-ciencia-tecnologia-e-innovacion/informe-general-2017

Lemus Maciel, B. (2018). Políticas en Ciencia y Tecnología en México 2006-2017. (Tesis de Doctorado). Universidad Nacional Autónoma de México.

Márquez Villegas, M. E. (2011). La ciencia en la UNAM. Modelos, políticas y desafíos para la medición del capital cultural en el Subsistema de la investigación Científica. (Tesis inédita de Doctorado). Universidad Nacional Autónoma de México, México.

Molina, A., Pérez, R., Bustos, E., Castaño, C., Jardey, O., \& Sánchez, M. (2013). Mapeamiento Informacional Bibliográfico de Enfoques y Campos Temáticos de la diversidad cultural: el caso de las revistas CSSE, Sci Edu. And Sci \& Edu. IX Encontro Nacional de Pesquisa em Educação em Ciências.

OEI. (2018). El estado de la ciencia. Principales indicadores de Ciencias y tecnología iberoamericanos e interamericanos. Buenos Aires: Altuna Impresores S.R.L. Recuperado de http://www.ricyt.org/wp-content/uploads/2018/10/www.ricyt_.org_files_edlc_2018.pdf

Pacheco Méndez, T. (2009). La transformación de la universidad y la formación para la investigación. En T. Pacheco Méndez, \& Á. Díaz-Barriga, Posgrado en educación (págs. 1747). México: Pensamiento Universitario 103.

Pacheco Méndez, T. (1994). Profesionalización de la universidad mexicana y de la investigación. En T. Pacheco Méndez, La organización de la actividad científica en la UNAM (págs. 61- 98). México: Librero Editor. 
Pérez Mora, R., \& Inguanzo Arias, B. (2018). La movilización del conocimiento en las políticas científicas en México. Horizontes Sociológicos, 69-81. Recuperado de https://www.researchgate.net/publication/326450487_La_movilizacion_del_conocimiento_e n_las_politicas_cientificas_en_Mexico

Pérez Mora, R., Prieto Quezada, M. T., \& Castellanos Gutiérrez, J. A. (2012). Las condiciones de producción intelectual de los académicos. Prolegómenos epistémico-metodológicos hacia la construcción de un objeto de estudio. En J. Naidorf, R. Pérez Mora, J. Naidorf, \& R. Pérez Mora (Edits.), Las condiciones de Producción intelectual de los académicos en Argentina, Brasil y México (págs. 13-32). Buenos Aires: Miño y Dávila.

Valero Olmedo, C., Molina García, A., \& Ponce Crespo, C. I. (2019). Políticas de ciencia, tecnología e innovación en México: aproximación a su análisis. Edähi Boletín Científico de Ciencias Sociales y Humanidades del ICSHu, 8(15), 65-72. Recuperado de https://repository.uaeh.edu.mx/revistas/index.php/icshu/article/view/5256/6855

Zepeda Gil, R. (2016). El juego de la asignación presupuestal a las universidades públicas estatales en México después de la transición democrática. Revisata Mexicada de Investigación Educativa, 21(70), 901-928. $\quad$ Recuperado de http://www.scielo.org.mx/pdf/rmie/v21n70/1405-6666-rmie-21-70-00901.pdf 